This is an electronic reprint of the original article. This reprint may differ from the original in pagination and typographic detail.

Author(s): Jantunen, Tommi; Mesch, Johanna; Puupponen, Anna; Laaksonen, Jorma

Title: $\quad$ On the rhythm of head movements in Finnish and Swedish Sign Language sentences

Year: $\quad 2016$

Version:

Please cite the original version:

Jantunen, T., Mesch, J., Puupponen, A., \& Laaksonen, J. (2016). On the rhythm of head movements in Finnish and Swedish Sign Language sentences. In J. Barnes, A. Brugos, S. Shattuck-Hufnagel, \& N. Veilleux (Eds.), Speech Prosody 2016 : Proceedings of the 8th International Conference on Speech Prosody, Boston University, USA, 31 May - 3 June 2016 (pp. 850-853). International Speech Communication Association. Speech prosody. https://doi.org/10.21437/SpeechProsody.2016-174

All material supplied via JYX is protected by copyright and other intellectual property rights, and duplication or sale of all or part of any of the repository collections is not permitted, except that material may be duplicated by you for your research use or educational purposes in electronic or print form. You must obtain permission for any other use. Electronic or print copies may not be offered, whether for sale or otherwise to anyone who is not an authorised user. 


\title{
On the rhythm of head movements in Finnish and Swedish Sign Language sentences
}

\author{
Tommi Jantunen ${ }^{1}$, Johanna Mesch ${ }^{2}$, Anna Puupponen ${ }^{1}$, Jorma Laaksonen ${ }^{3}$ \\ ${ }^{1}$ University of Jyvaskyla, Department of Languages, P.O. Box 35, FI-40014 University of \\ Jyvaskyla, Finland. \\ ${ }^{2}$ Stockholm University, Department of Linguistics, SE-106 91 Stockholm, Sweden. \\ ${ }^{3}$ Aalto University, Department of Computer Science, P.O. Box 15400, FI-00076 Aalto, Finland. \\ tommi.j.jantunen@jyu.fi, johanna.mesch@ling.su.se, anna.puupponen@jyu.fi, \\ jorma. laaksonendaalto.fi
}

\begin{abstract}
This paper investigates, with the help of computer-vision technology, the similarities and differences in the rhythm of the movements of the head in sentences in Finnish (FinSL) and Swedish Sign Language (SSL). The results show that the movement of the head in the two languages is often very similar: in both languages, the instances when the movement of the head changes direction were distributed similarly with regard to clause-boundaries, and the contours of the roll (tilting-like) motion of the head during the sentences were similar. Concerning differences, direction changes were found to be used more effectively in the marking of clause-boundaries in FinSL, and in SSL the head moved nearly twice as fast as in FinSL. However, the small amount of data means that the results can be considered to be only preliminary. The paper indicates the roll angle of the head as a domain for further work on headrelated rhythm.
\end{abstract}

Index Terms: rhythm, head movement, sentence, Finnish Sign Language, Swedish Sign Language

\section{Introduction}

In this paper we investigate the similarities and differences in the rhythm of movements of the head in sentences of two historically related sign languages, Finnish Sign Language (FinSL) and Swedish Sign Language (SSL). We define the notion of rhythm as 'the organization of units in time' and presume that the rhythmic feel of a sentence, as of any linguistic unit, is determined by the linearly occurring phonetic properties and events that are used in the marking of the inner and outer borders of the sentence (i.e. clause-boundaries) and its area (see [1], [2]). In previous studies (e.g. [3], [4], [5]), it has been suggested that the markers of rhythmic sequences in signed language are, for example, punctual indices (e.g. a single change in the position of the hands or head during a relatively short period of time) and articulatory contours (e.g. the series of changes in the position of the hands and head during a longer stretch of time). Accordingly, we approach the rhythm of head movements in FinSL and SSL sentences with two main research questions: (i) How do the moments when the movement of the head changes direction contribute to the marking of clause-boundaries in FinSL and SSL, and (ii) How are the movements of the head distributed in terms of their amplitude in FinSL and SSL sentences (amplitude is here understood in its general sense)? If the results show valid differ- ences between FinSL and SSL, we consider the rhythm of the movement of the head in the sentences of the two languages to be different; otherwise, we interpret the rhythm to be similar in terms of the investigated parameters.

The focus of the paper on the rhythm of head movements (instead of, for example, on the movement of the hands) derives from the fact that the present work is part of two larger on-going projects investigating both nonmanuality (i.e. the activity of other articulators than the hands, especially the head) and rhythm in sign languages (see [5], [6]). The nonmanual behavior of signers has been increasingly researched in recent decades, but at the moment little is known about the rhythm of sign languages (e.g. [3], [4], [7], [8]), let alone the rhythm of sign language sentences (see [4], [9]). However, native signers usually have an inner feel of the rhythm of signing which they often refer to especially when asked to describe the differences between sign languages [6]. Native signers have also reported the existence of such a difference between FinSL and SSL, and this observation functions as the main motivator for the comparative aspect of the present study.

\section{Data and methodology}

The study is based on 16 declarative sentences ( 8 for FinSL and 8 for SSL) produced by $8(4+4)$ signers. All the sentences have been extracted from longer narratives telling the Snowman story, in which the sentences occurred story-initially. Syntactically, the productions of each signer corresponded to coordinated two-sentence sequences and, semantically, all the sequences expressed exactly the same contents (roughly: 'the boy wakes up and sees that it is snowing outside'). The relatively small size of the data is explained both by the piloting nature of the present work and the practical difficulties of finding syntactically and semantically commensurable (and thus comparable) sentences in both languages.

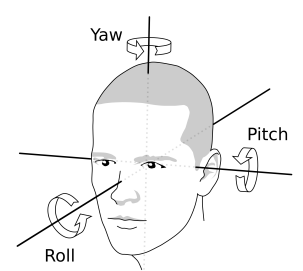

Figure 1: The yaw, pitch, and roll angles in the human head motion [11]. 


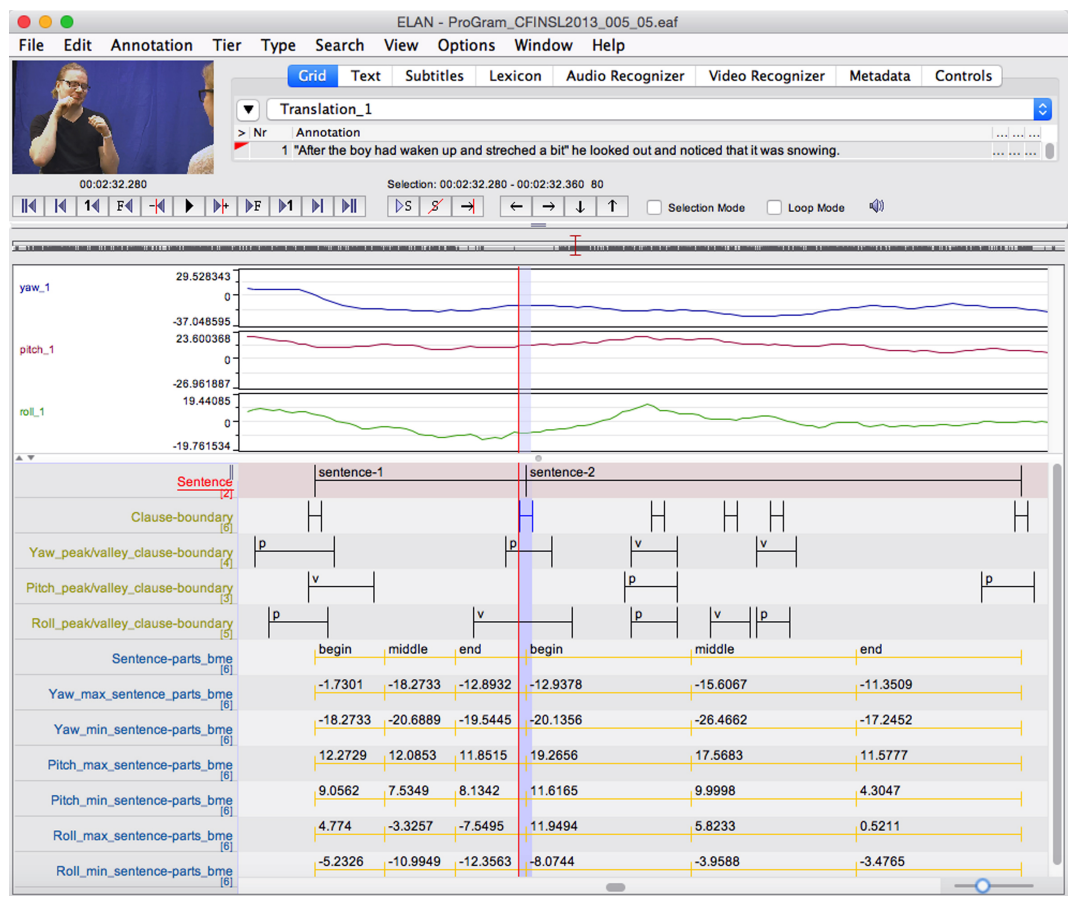

Figure 2: FinSL data in ELAN showing a) visual descriptors of the yaw, pitch, and roll angles generated with SLMotion, b) human made sentence, clause-boundary and peak/valley on clause-boundary annotations, and c) maximum and minimum values (calculated in ELAN) for the three sentence parts in yaw, pitch, and roll dimensions (used in the calculation of $R$ ).

All the stories have been annotated in ELAN [10] for signs, sentences and head movements (https://tla.mpi.nl/tools/tlatools/elan/). The research material also includes 3D numerical data on the head movements of signers describing the motion of the head in the yaw, pitch, and roll dimensions (i.e. a turning-like motion, nodding-like motion, and tilting-like motion, respectively; see Figure 1). The 3D data has been obtained with computer-vision technology [11] implemented in SLMotion software [12] (http://research.ics.aalto.fi/cbir/software/ slmotion/). The present study could not have been done without SLMotion although in general the background technology is not yet ready for the automatic detection and large scale quantitative analysis of head position angles.

In order to answer the first research question, clause-level boundaries were first determined visually by the researchers for each sentence by following the view on the clause presented in [13]. In both languages, the total number of clauseboundaries was 26 , the number of boundaries per signer being either six or seven. After this, the visual yaw, pitch, and roll descriptors of the 3D data were compared qualitatively in ELAN with the clause-boundary annotations (see Figure 2). The instances when the head changed direction were equated with major visual peak and valley shapes of the three descriptors. If a peak or a valley shape of any of the descriptors was observed to occur simultaneously with a clause-boundary annotation, the boundary was counted as being associated with an instance when the movement of the head changed direction. The total number of these instances in all three dimensions was also counted for each sequence.

For the second research question, each of the 16 sentences was divided automatically in ELAN into three sub-sequences (the opening, middle, and closing). After this, the numerical range $(\mathrm{R})$ of the amplitude of the head motion for yaw, pitch and roll dimensions was calculated for each sub-sequence on the basis of the highest and lowest yaw, pitch and roll value in each sub-sequence. In addition to indicating the largest amplitude of the movement executed by the head during each subsequence, the range values - when inspected in the function of time - also indicate the speed of the movement of the head. Signer-specific range values were employed to calculate language-specific averages for the change in the amplitude of the head movement in the sentences. The similarities and differences between the two languages were investigated both qualitatively (via visual observation) and with the linear correlation co-efficient (r).

\section{Results}

\subsection{How the instances when the head changes direc- tion contribute to the marking of clause-boundaries in FinSL and SSL}

In both FinSL and SSL, the relative percentage of clauseboundaries that were associated with an instance when the movement of the head changed direction in any of the three dimensions was, on average, 65.5 percent: in FinSL, the share of clause-boundaries marked this way was 64 percent $(\mathrm{n}=50 / 78$, i.e. the total number of hits in all three dimensions over the total number of clause-boundaries in all three dimensions) and in SSL it was 67 percent $(n=52 / 78)$. In other words, the signers of both languages similarly and quite often were found to associate clause-boundaries with instances when the movement of the head changed direction. Moreover, as seen from Table 1, none of the three dimensions (yaw, pitch, roll) were particularly favored or avoided in the marking: only movement direction changes of the head in the yaw dimension were slightly more frequent in SSL than in FinSL, but this is 
explained by the way one signer in each language moved their head: with one FinSL signer the frequency was only 33 percent and with one SSL signer it was a full 100 percent, the rest of the signers in both languages performing near 70 percent. One point that emerged clearly from the data was that the sentence boundaries were always layered with a direction change in the movement of the head in some dimension.

Table 1. The absolute and relative percentage of clause-boundaries associated with a moment when the movement of the head changes direction in the productions of four FinSL and four SSL signers.

\begin{tabular}{|cc|c|c|c|}
\hline \multicolumn{1}{c}{} & \multicolumn{2}{c}{ FinSL } & \multicolumn{2}{c|}{ SSL } \\
& abs. & rel. & abs. & rel. \\
\cline { 2 - 5 } yaw & $16 / 26$ & $62 \%$ & $20 / 26$ & $77 \%$ \\
pitch & $17 / 26$ & $65 \%$ & $16 / 26$ & $62 \%$ \\
roll & $17 / 26$ & $65 \%$ & $16 / 26$ & $62 \%$ \\
\hline
\end{tabular}

However, only an average of 32.5 percent of all the instances when the movement of the head changed direction in the investigated sequences occurred during a clause-boundary: in FinSL, the share was 35 percent $(n=50 / 142$, i.e. the number of instances of a head movement direction change associated with a clause-boundary in all three dimensions over the total number of instances of a head movement direction change in all three dimensions) and in SSL it was 30 percent $(n=52 / 172)$. In other words, the signers of the two languages behaved fairly similarly also in not moving their heads only for the purpose of marking clause-boundaries. Table 2 shows the results for the yaw, pitch, and roll dimensions. From the table it can be seen that there is a relatively large difference between the two languages in the use of the head movement direction change in the roll dimension: the direction changes in this dimension were used more effectively to mark clause-boundaries in FinSL than in SSL. This difference cannot be explained simply by the behavior of any individual signer.

Table 2. The absolute and relative percentage of those direction change instances in the movement of the head that were associated with a clause-boundary in the productions of four FinSL and four SSL signers.

\begin{tabular}{|cc|c|c|c|}
\hline \multicolumn{1}{c}{} & \multicolumn{2}{c}{ FinSL } & \multicolumn{2}{c|}{ SSL } \\
& abs. & rel. & abs. & rel. \\
\cline { 2 - 5 } yaw & $16 / 48$ & $33 \%$ & $20 / 50$ & $40 \%$ \\
pitch & $17 / 52$ & $33 \%$ & $16 / 60$ & $27 \%$ \\
roll & $17 / 42$ & $40 \%$ & $16 / 62$ & $26 \%$ \\
\hline
\end{tabular}

\subsection{How the movement of the head is distributed in terms of its amplitude in FinSL and SSL sentences}

The results of the second research question - summarized in Figure 3 - show both similarities and differences. A notable similarity in the results for the two languages was the extent to which in both FinSL and SSL the amplitude of the movement of the head in the roll dimension decreased toward the end of sentences $(r=1.0$, i.e. a perfect linear positive correlation). In other words, in both languages, the tilting-like movement of the head was larger in the opening section of sentences than in the closing section. Moreover, when examined in the function
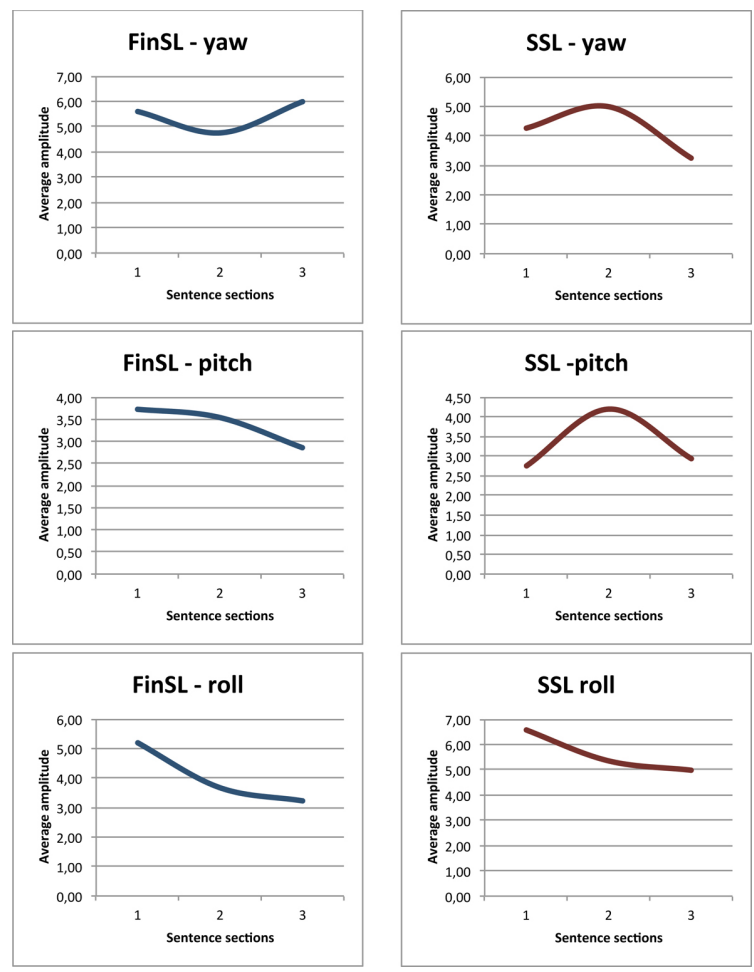

Figure 3: The average amplitude (in degrees) of the head in yaw, pitch, and roll dimensions for the opening (1), middle (2), and closing (3) sections of $8+8$ semantically and structurally comparable FinSL and SSL sentences.

of time, the results also indicate that in both languages the speed of the movement of the head in the roll dimension was very similar.

In the yaw and pitch dimensions the results show big differences between FinSL and SSL. In the yaw dimension, the shapes of the language-specific amplitude curves of the movement of the head were almost diametrically opposed to each other $(\mathrm{r}=-0.95$, i.e. a near perfect negative correlation) and in the pitch dimension the shapes of the curves were simply different, showing no linear correlation at all $(\mathrm{r}=0.19)$. These differences were also reflected in the speed of the head movement: in FinSL, the head only moved at approximately half of the speed of the head movements in SSL; that is, Swedish signers moved their heads at almost double the speed of FinSL signers.

\section{Discussion}

In terms of the first research question, we consider the results were fairly predictable, as syntactic borders in many sign languages have often been reported to be marked by a direction change in nonmanual activity (e.g. [4], [14]). Thus, the general finding that FinSL and SSL tend to align the moments when the movement of the head changes direction with clauseborders at a similar frequency is not surprising. However, the results of the first question also indicate a potential rhythmic difference between FinSL and SSL in the degree to which the languages employ the head movement direction change in the roll dimension to mark clause-boundaries (see Table 2): the fact that FinSL signers associated the direction change moments in the roll dimension with clause-boundaries more ef- 
fectively than SSL signers did may imply a deeper underlying rhythmic difference between FinSL and SSL. However, because of the small quantity of data in this study, we do not yet want to draw any final conclusions on the issue.

Concerning the second question, the results show that there are more differences (cf. the yaw and pitch dimensions) than similarities (the roll dimension) in the rhythmic movements of the head in FinSL and SSL sentences. However, as far as the differences are concerned, we are not yet sure if they are genuine examples of language-wide rhythmic differences: it is possible that they are simply the result of the individual variation present in the data, or even deviations caused by the head tracking technology. Concerning the latter point we know, for example, that the 3D movement of the head particularly in the pitch dimension cannot be captured as effectively from the video as the movement of the head in the other two dimensions [11]. Consequently, more research into these differences and their relationship to rhythm is needed.

As far as the similarities in the answers to question two are concerned, we find it very interesting that in both languages the movement of the head in the roll dimension was larger in the early parts of sentences than in the final parts. This may indicate that, in the roll dimension, there is a rhythmic similarity between the two languages - a fact that has not been previously attested for FinSL and SSL. However, having said this, we still want to emphasize that this result too is very preliminary and should be treated at this point as no more than a hypothesis to be tested in the future.

On a more general level, we interpret the results of both questions together as suggesting that it is perhaps particularly the roll dimension of the head movement that expresses the most profound rhythmic similarities and differences between FinSL and SSL sentences. This favoring of the roll dimension in the expression of rhythm can perhaps be explained functionally. For example, it is our hypothesis that the head movement in the yaw and pitch dimensions is used as a structural part of lexical signs more often than the movement in the roll dimension (e.g. EI-TYKKÄ̈̈ 'does not like', ONKUULLUT 'have heard' for FinSL; OMÖJLIGT 'impossible', VETA*PERF 'know already' for SSL). Moreover, it is obvious that many elementary linguistic functions in sign languages (e.g. negation, affirmation, and emphasis) are typically expressed along the yaw and pitch dimensions, not along the roll dimension [5]. Consequently, we suggest that in future work on the rhythm of head movements in sign languages the roll dimension should be given particular attention.

\section{Conclusions}

In this paper we have investigated similarities and differences in the rhythm of the movement of the head in sentences in Finnish and Swedish Sign Language, using computer-vision technology. The investigation revealed that in many cases the rhythmic movement of the head is very similar in the signers of both languages, yet differences can also be found. Given the relatively small amount of data used in the present work, we have hesitated to generalize the results as true instances of language-wide examples of rhythmic similarities and differences. However, on the basis of the data and results, we propose that the movement of the head especially in the roll dimension might be important in manifesting these rhythmic similarities and differences.
Owing to its connection to projects that are still ongoing, this study has focused only on the activity of the head. However, the head is only one articulator involved in the creation of linguistic messages in sign languages, which means that the feel of a rhythmic difference between FinSL and SSL, reported by native signers, may just as well be caused by the activity of other articulators. In future work on rhythm, we will deal more with the activity of these other articulators too.

\section{Acknowledgements}

The authors wish to thank the Academy of Finland for its financial support under grants 269089 \& 273408 for ProGram, 140245 for CoBaSiL, and 251170 for COIN. The authors also gratefully acknowledge the financial support of Emil Aaltonen Foundation, and Riksbankens Jubileumsfond (grant In20080276-1-IK for Korpus för det svenska teckenspråket).

\section{References}

[1] A. D. Patel and J. R. Daniele, "An empirical comparison of rhythm in language and music," Cognition, vol. 87, pp. B35B45, 2003

[2] A. D. Patel, "Musical rhythm, linguistic rhythm, and human evolution," Music Perception, vol. 24, no. 1, pp. 99-104, 2006.

[3] P. Boyes Braem, "Rhythmic temporal patterns in the signing of deaf early and late learners of Swiss German Sign Language," Language and Speech, vol. 42, no. 2-3, pp. 177-208, 1999.

[4] W. Sandler, "Visual prosody," in R. Pfau, M.| teinbach and B. Woll (eds.), Sign language: An international handbook. Berlin: Mouton De Gruyter, pp. 55-76, 2012.

[5] A. Puupponen, T. Wainio, B. Burger and T. Jantunen, "Head movements in Finnish Sign Language on the basis of Motion Capture data: A study of the form and function of nods, nodding, head thrusts, and head pulls," Sign Language \& Linguistics, vol. 18 , no. 1 , pp. 41-89, 2015.

[6] T. Jantunen, J. Mesch and A. Puupponen, "Aspects of rhythm in Finnish and Swedish Sign Language," a paper to be presented in the 12th Conference on Theoretical Issues in Sign Language Research (TISLR 12), Melbourne, Australia, 4-7 January, 2016.

[7] R. B. Wilbur and S. B. Nolen, "The duration of syllables in American Sign Language," Language and Speech, vol. 29, no. 3, pp. 263-280, 1986.

[8] G. Allen, R. B. Wilbur and B. Schick, "Aspects of the rhythm in ASL," Sign Language Studies, vol. 72, pp. 297-320, 1991.

[9] T. Jantunen, "Rinnastuksen prosodiaa suomalaisessa viittomakielessä" [The prosody of clausal coordination in FinSL], in M. Lehtinen and U. K. Laine (eds.), XXIX Fonetiikan päivät, Espoo 20.-21.3.2015, Julkaisut - Papers. Helsinki: Aalto University, pp. 15-21, 2015.

[10]O. Crasborn and H. Sloetjes, "Enhanced ELAN functionality for sign language corpora," in O. Crasborn, E. Efthimiou, T. Hanke, E. D. Thoutenhoofd and I . Zwitserlood (eds.), Construction and exploitation of sign language corpora. Proceedings of 3rd Workshop on the Representation and Processing of Sign Languages at LREC 2008. Paris: ELRA, pp. 39-43, 2008.

[11] M. Luzardo, M. Karppa, J. Laaksonen, T. Jantunen, "Head pose estimation for sign language video," in J.-K. Kamarainen and M. Koskela (eds.), Image Analysis. Springer, Lecture Notes in Computer Science, Vol. 7944, pp. 349-360, 2013.

[12] M. Karppa, V. Viitaniemi, M. Luzardo, J. Laaksonen and T. Jantunen, "SLMotion - An extensible sign language oriented video analysis tool," in Proceedings of LREC 2014. Paris: ELRA, pp. 1886-1891, 2014.

[13] T. Jantunen, "Ellipsis in Finnish Sign Language," Nordic Journal of Linguistics, vol. 36, no. 3, pp. 303-332, 2013.

[14] R. Pfau and J. Quer, "Nonmanuals: Their prosodic and grammatical roles," in D. Brentari (ed.), Sign Languages: A Cambridge language survey. Cambridge: Cambridge University Press, pp. 381-402, 2010. 